Geograficando, vol. 14, n. ${ }^{\circ}$ 1, e038, junio 2018. ISSN 2346-898X

Universidad Nacional de La Plata.

Facultad de Humanidades y Ciencias de la Educación.

Departamento de Geografía

\title{
Entrevista a Sergio García García
}

\section{Mariana Relli}

Diplomado en Trabajo Social, Licenciado en Sociología y Doctor en Antropología. Docente del Departamento de Trabajo Social y Servicios Sociales de la Facultad de Trabajo Social de la Universidad Complutense de Madrid. Integrante del Colectivo Carabancheleando.

E: entrevistadora Mg. Mariana Relli

SGG: Sergio García García

Cita sugerida: Relli, M. y García García, S. (2018). Entrevista a Sergio García García. Geograficando 14 (1), e038. https://doi.org/10.24215/2346898Xe038 


\section{Entrevista a Sergio García García.}

Diplomado en Trabajo Social, Licenciado en Sociología y Doctor en Antropología. Docente del Departamento de Trabajo Social y Servicios Sociales de la Facultad de Trabajo Social de la Universidad Complutense de Madrid. Integrante del Colectivo Carabancheleando.

E: entrevistadora Mg. Mariana Relli

SGG: Sergio García García

\section{E: Desde Geograficando nos acercamos a usted por el interés que nos despertó el trabajo del Colectivo Carabancheleando en tanto ámbito de construcción de conocimientos entre actores diversos ¿podría comentarnos de quése trata esta experiencia?}

SGG:En el año 2012, desde el Observatorio Metropolitano de Madrid, se inició una investigación dedicada a explorar el impacto de la crisis sobre las periferias madrileñas.Se partió de la hipótesis de que la ofensiva neoliberal estaba construyendo un nuevo modelo social que redundaba en la caída de las clases medias y en el resurgimiento del fantasma de las periferias. Comenzamos recorriendo barrios en lo que llamamos paseos $o$ derivas con el fin de adentrarnos en sus dinámicas y, con el correr de los meses nos fuimos encontrando con movimientos sociales y vecinales que surgían o resurgían al calor del Movimiento 15-M, de gran efervescencia en el año 2013.

El barrio de Carabanchel, un distrito de 250.000 habitantes que contiene toda la heterogeneidad de la periferia madrileña, se convirtió en un ámbito privilegiado para observar las consecuencias de la crisis, las dinámicas barriales y las resistencias. La Asamblea de Vecinos y Vecinas de Carabanchel fue una de las más activas, y se puso al frente de las luchas contra los desahucios y las redadas policiales a la población migrante, de gran presencia en el barrio. Desde la Asamblea se ocupó un edificio abandonado y se levantó el Centro Social EKO, espacio de encuentro de colectivos diversos y actividades barriales en las que la autogestión es central. En ese movimiento, inmerso en un contexto de florecimiento de actividades culturales y contraculturales en el barrio (música, teatro, viviendas colectivas, huertos urbanos en solares desocupados, etc.), y con el acercamiento de la propuesta del Observatorio Metropolitano de Madrid, nació Carabancheleando, que está integrado por personas y colectivos de diferentes trayectorias vitales, educativas, laborales y militantes.

Armamos una propuesta de investigación militante que se basa en pensar con otros y otras, desde lo colectivo, para respondernos a las preguntas sobre qué está pasando, qué nos está pasando, qué podemos hacer frente a ello. De alguna manera, la propuesta se enfrenta tanto a los saberes académicos, distanciados de las vivencias cotidianas en los ámbitos que aparecen como recortes de objetos de estudio, como al ombliguismo de la militancia barrial, muchas veces encerrada en sí misma sin interés por el contexto social y sus problemas. Intentamos superar esas limitaciones a partir del reconocimiento y la producción de saberes subalternos, que en nuestro caso llamamos saberes periféricos.

\section{E: ¿Cómo definen a la periferia? ¿Qué hace que un barrio sea considerado de la periferia?}

SGG:La posición geográfica de un barrio no lo define como periférico por sí misma. Los alrededores de Madrid presentan distritos con diferencias muy marcadas, aun a similares distancias del centro. A la periferia se la asocia con problemas sociales, carencias de todo tipo, eso no sucede en todos los barrios que, geográficamente, podrían definirse como periféricos. 
Las periferias, en el sentido en que las entendemos nosotros, son ámbitos donde ha operado la política habitacional desde la década del 50, donde fueron realojadas familias que habitaban en chabolas y se generaron concentraciones de población estratificada según sus niveles de renta, con fuertes consecuencias en la segregación social. La presencia o ausencia de recursos comerciales y sociales (educativos, sanitarios, de transporte, de limpieza) también caracterizan a la periferia. Esos elementos, junto a la presencia policial permanente bajo la forma de controles públicos, contribuyeron a la creación de una estigmatización social que cae sobre estos barrios y es reforzada aun por personas que nunca han transitado por ellos. La sedimentación histórica de la pobreza, el abandono institucional y el estigma acaban incidiendo en lo que hemos llamado el "miedo ambiente" de los barrios periféricos, con sus territorios solitarios u hostiles y su permanente monitoreo por parte del Estado.

Los elementos fundamentales en la construcción de la periferia constituyen un puchero que mezcla realojos, distancia al centro, recursos escasos, miedos, abandonos institucionales, marcas y, sobre todo, desigualdad hacia adentro y hacia afuera. Pero por supuesto hay más cosas, milagros cotidianos que pueblan estos barrios, redes de solidaridad, mucha calle, inteligencia, respeto, estrategias de personas sistemáticamente excluidas que despliegan fuerzas y formas alternativas de vivir en sociedad.

\section{$\mathrm{E}:$ ¿Cómo definen a los saberes periféricos?}

SGG:Llamamos saberes periféricos a las formas de conocimiento que se generan en las periferias: periferias en sentido geográfico, los barrios empobrecidos alejados de los centros económicos y de poder, y periferias en sentido social, aquellos espacios cuya cultura es descalificada o invisibilizada.

Los saberes periféricos tienen un modo propio de usar el lenguaje (con vocabulario y entonaciones propias), tienen significados propios de las cosas (por ejemplo, la vivienda no significa lo mismo en Las Rozas, barrio periférico de sectores medio-altos y altos, que en Carabanchel), e incluyen también saberes prácticos, es decir, formas de comportarse en el espacio público, de sobrevivir a las situaciones difíciles, que no se observan en otros ámbitos geográficos ni sociales.

Rescatamos esos saberes periféricos porque entendemos que el saber siempre tiene que ver con el poder. Para ejercer influencia sobre el mundo, hace falta dotarse de un saber sobre ese mundo que oriente la acción. Pero además, cualquier forma de poder siempre instaura formas de saber: lo que se enseña en la escuela o en los medios siempre es el relato que el poder necesita para sus intereses, por eso hace falta un saber propio, autónomo, desde las gentes de las periferias, que sirva a sus intereses. Esto es algo que el movimiento vecinal comprendió muy bien en su día, apuntando a visibilizar los problemas que se vivían a diario en las áreas que concentraban la mayor parte de la población de la ciudad, y que tradujeron en luchas por la dotación de equipamientos comunitarios, servicios de salud, escuelas, bibliotecas, transportes.

En las formas hegemónicas de saber, los barrios periféricos son, como decía, invisibilizados, desvalorizados, con estigmas negativos sobre su población que demuestran un total desconocimiento. También son folclorizados, sobre todo, en las antesalas de procesos de renovación urbana y gentrificación, y son siempre afectados por la mirada externa, la exterioridad, descritos y nombrados por quienes, desde afuera, "los estudian" (y realizan producciones que luego no revierten en esos mismos barrios, se quedan en la academia para alimentar currículums).

\section{$\mathrm{E}:$ ¿Con qué metodología de trabajo han abordado la producción de saberes periféricos?}

SGG:Comenzamos con encuentros de mapeo colectivo a los que se invitaba a miembros de organizaciones vecinales y sociales del distrito. Así conseguimos trazar una representación gráfica subjetiva que obedecía a nuestros propios intereses y los de las luchas del barrio. Allí aparecen los límites, las concentraciones de algunas problemáticas, la segregación interna, etc. Agrupamos al conjunto de cuestiones que fueron apareciendo en estos encuentros de mapeo colectivo en cuatro categorías: las fronteras (internas y externas, físicas y simbólicas, raciales, económicas), los estigmas (espacios y barrios), la crisis (y sus manifestaciones 
en la vivienda, el comercio, los servicios sociales, etc.) y los movimientos (vecinales, culturales y sociales). Luego organizamos diversos paseos y derivas a partir de esas cuatro categorías, con itinerarios y paradas en las cuales una o varias personas implicadas en el tema y en el lugar nos daban su visión, su explicación, sobre lo que había sucedido o estaba sucediendo en ese lugar. Y luego de esas exposiciones disparadoras se armaban conversaciones y se iba creando un discurso colectivo a partir de las relaciones significativas entre las experiencias previas, los conceptos, los lugares, las personas. Los paseos permiten andar y conversar al mismo tiempo, el paseo hablado es algo de la gente común, iguala saberes, es algo que podemos hacer todos y todas independientemente de nuestro capital cultural. Y realizar la reflexión sobre los espacios vivos sumaba acontecimientos no planificados (interacción con gente del barrio, sucesos inesperados, conflictos), lo que enriquecía la experiencia.

Se iban tomando registros de los paseos y se publicaban crónicas en la página web que, debido a la densidad de conocimiento que allí se generaba, sólo lograban rescatar partes de su riqueza. En un paseo de cuatro horas, en el que participan decenas de personas, se genera una densidad de conocimiento intersubjetivo equiparable a la de varios meses de investigación etnográfica. Gran parte de lo producido está en la página web y también fue semillero del Diccionario de las periferias.

\section{E: Hábleme sobre el Diccionario de las periferias...}

SGG:El Diccionario de las periferias comenzó a perfilarse a partir de las publicaciones que íbamos construyendo y colocando en la página web del proyecto. Ahora está publicado por la editorial cooperativa Traficantes de Sueños, se puede conseguir en papel o descargar gratuitamente de la página web.

La apuesta fue por el reconocimiento de saberes existentes y la producción de nuevos saberes autónomos. Pensamos que es una herramienta útil para disputar significados sobre nuestra realidad, reflejarla y también producirla, desde una reflexión colectiva que parta de la experiencia y del lenguaje propio, tal como se usa en los barrios. Apoyo mutuo, ascensor, bares, barrionalismo, buscar la vida, casa tapiada, comunes, comunidad, desabucio, descampados, curro, El Guille, institución, línea gris, litrona, mercadillo, necesidad, okupaciones, $P A U$, racismo, redadas racistas, segregación escolar, trabajo social e intervención social, trapicheo, vivienda colectiva, etc. son sólo algunas de las palabras cuyos significados recoge el Diccionario de las periferias, excusas para contar historias y hablar de las cosas de los barrios.

En el Diccionario intentamos dar voz a la diversidad de relatos que emergen de las periferias, se podrá ver que algunas palabras tienen más de un significado, puesto que en el proceso de construcción del mismo no hubo acuerdos o aparecieron distintos usos que, en pos de valorizar el proceso, se incluyeron en su totalidad.

Pero en el andar también aparecieron otras propuestas, como la producción del audiovisual $K$-de-Kalle junto con el grupo Cine sin Autor.

$K$-de-Kalle surgió de un encuentro con chavales que hacían hip hop en la zona de Caño Roto, que conocimos precisamente en uno de los paseos que hicimos al principio del proceso, concretamente en el paseo de "estigmas". A través del contacto con la Asociación de Vecinos surgió la posibilidad de conocer a estos chavales, que nos dieron un paseo por el barrio. Nos gustó tanto su visión y su forma de estar arraigados al barrio, y de al mismo tiempo tener una mirada reflexiva sobre el mismo, que les propusimos hacer algo juntos, que fuese parte de esa investigación sobre las periferias, pero con un formato y un método que no fuese como el que habíamos hecho hasta ese momento, sino que estuviese adaptado también a sus deseos. Y como ellos tenían un estudio de hip hop, dijeron que les apetecía hacer un videoclip y lo que hicimos, en contacto con otro colectivo de cine asambleario que se llama Cine Sin Autor, pues, es hacer durante un verano un proceso de conocimiento del barrio con el fin de grabar el videoclip. Ese proceso se quedó a medias, porque no se acabó el producto finalmente, por las precariedades vitales de estos chavales, pero sí que fue una experiencia muy bonita, muy chula, para todos los que allí estábamos implicados.

E: Luego del camino transitado por Carabancheleando y teniendo en cuenta la inserción laboral de alguno/as miembros del colectivo en la Universidad, ¿han construido vínculos con la academia? La producción de saberes 
periféricos ¿ha podido permear la actividad académica, tanto en su desempeño como investigadorelas y docentes? ¿Han logrado despertar el interés de otro/as colegas y/o estudiantes de la institución en la que trabajan?

SGG:En Carabancheleando siempre ha habido como una especie de autocontrol, auto responsabilización por parte de quienes estábamos, en el sentido de no traer lógicas, tips, lenguajes académicos a este proceso, a pesar de que algunos y algunas estábamos muy vinculados laboral y personalmente, o porque estábamos haciendo investigaciones, en el mundo académico. Aun así, lógicamente, al estar insertos en ese mundo, por los propios contactos personales y luego por el interés que ha despertado alguna de las cosas que hemos hecho, como el Diccionario, sí que es verdad que desde el mundo académico se nos ha interpelado para participar en clases de másteres dedicados a temas urbanos o a temas de metodologías comunitarias, a participar en algunos textos, en algunos libros, ya no solamente desde el mundo académico, sino también desde otros espacios de generación de conocimiento y de saber por fuera de la academia, pero que de algún modo utilizan conocimiento experto.

¿Cómo lo hemos usado dentro del mundo de la academia quienes estamos en Carabancheleando? Por ejemplo, algunos y algunas somos profesores en la universidad y en educación secundaria y hemos tratado de trasladar el trabajo del Diccionario a nuestras aulas, dándolo a conocer porque algunas palabras o el Diccionario en sí se vinculaba con temas que abordamos en nuestras asignaturas, o bien como excusa para impulsar dentro de nuestras aulas un conocimiento autónomo por parte del propio estudiantado, que le permita hacerse dueño del conocimiento sobre sus propios entornos sin necesidad de depender del conocimiento de otros. Hemos hecho actividades para que elaboren entradas de diccionario,y también hemos utilizado la metodología de los paseos y los mapeos para trabajar en clase, dependiendo de las temáticas.

Y en cuanto a los resultados en sí de esta investigación colectiva, que no es a lo que más importancia le damos, sino más al proceso, pero sí que algunas cosas ha dado como fruto, destacar que gente que está realizando investigaciones en la universidad, en el campo de la sociología urbana, se han servido de algunas de las categorizaciones que hemos realizado sobre la periferia en el Estado español y específicamente en Madrid. El otro día nos contaban, por ejemplo, que esa categorización de los distintos tipos de periferia que se habían ido sedimentando en Madrid y que nosotros habíamos registrado en el Diccionario y en otros textos, pues a ellos les salía prácticamente calcada a partir del estudio de datos sobre población en los distintos barrios. Desde ahí sí que ha habido una cierta transferencia de conocimientos, o por lo menos comunicación y sintonía con otros grupos de investigación de temas urbanos insertos en lo académico.

E y SGG: Extrajimos del Diccionario de las Periferias tres definiciones que ilustran los distintos lenguajes y la vocación por evocar de una manera para nada romántica las construcciones conceptuales que se abordaron en el proyecto. Al final, están los enlaces a los sitios web, como una invitación a seguir leyendo...

\section{BARRIONALISMO}

"Sí señor, Satánico y de Carabanchel”afirmaba en la mítica película El Día de la Bestia un heavy carabanchelero de pro, justo de ese mismo barrio (pero con k) al que el colectivo SlumWear se refiere con orgullo como la "Universidad de la Calle". Vallekas Mola es lo que rezan muchas pegatinas adheridas a cristales de coches que, un día al año, ceden su espacio a piratas que celebran que Vallekas es Puerto de Mar (los bukaneros del Rayo descansan en agosto). Igual de veloces se agotaron en las fiestas del barrio las camisetas que en todos los colores imaginables reclamaban que "Aluche isnotSpain". Cerca, Black Bee le canta a su Villaverde City "el barrio de los quinquis, porque somos quinquis", mientras que el mismo distrito, otra crew de San Cristóbal (SkyleeCrew) invita a la "Hood Nation, (a) alzar la mano cabrón: Defiende tu barrio y demuestra tu son".

Barrionalismo es eso y mucho más. Es el sentimiento de orgullo y pertenencia con el que muchos de los habitantes de las periferias responden a la estigmatización y marginación que sufren sus barrios. Funciona como un punto fuerte de identidad y de construcción de una experiencia común: el barrio es ese lugar 
donde todo el mundo se reconoce, habla el mismo idioma, donde todos tienen problemas parecidos, independientemente del lugar del que procedan, donde es posible hacer comunidad y pelear juntos por un mundo mejor. Y eso, bien merece alzar la cabeza, a pesar de que la desigualdad se palpe en cada esquina de estos barrios bajos. El Barrio -con mayúsculas-, donde vive "mi peña", cuna de resistencias, territorio de vida y lema... lugar en el que quedarse "aunque me haga rico".

A veces, sin embargo, este sentimiento barrionalista se torna peligrosamente excluyente.Sucede cuando, como en todo nacionalismo, se exige pureza de sangre para poder reclamar ese sentimiento de pertenencia (¿puede un migrante ser barrionalista? ¿Cuántas pruebas se requieren para demostrar ser un carabanchelero legítimo?), o cuando, nuevamente al igual que en demasiados “-ismos", se idealiza una historia, una época dorada del pasado que se contrasta con un presente degradado por culpa de algunos de los nuevos habitantes. Aquí el barrionalismo deja de ser prometedor y se convierte en más de lo mismo.

\section{Curro}

Es algo bipolar, con sus cosas positivas y negativas. Nosotras somos más del barrio que los columpios así que alguna vez lo hemos llamado trabajo, pero vamos, que nosotras no vamos a nuestro "empleo remunerado", vamos al "curro". Es algo más de barrio, más duro, barriobajero, macarra. Tú no oyes a un pijo decir "voy al curro", en todo caso van al "Job" o al "work", y si dicen curro es porque se agobian cuando tienen demasiado trabajo.

Nosotras, por ejemplo, somos currelas, somos unas currantas. Nosotras no decimos "ese es un trabajador de puta madre", nosotras decimos "ese es un currante" o "cómo te lo has currao". Una puede decir que una persona trabaja muy bien, pero esa, en cambio, curra que te cagas. Es algo distinto, tiene algo más... algo que marca la diferencia.

El curro requiere esfuerzo, hacer las cosas con dos cojones, pam, pam, pam!!! Vamos, que no levantas cabeza. Es un rollo, es duro y estresante, vamos, que es una mierda pinchá en un palo. Es una obligación, algo que hacemos para sobrevivir, alcanzar unas metas y gastarnos el dinero en lo que queramos. También tiene sus rutinas, de lunes a viernes, su horario de 8 a 15hs, sus reglas, sus normas y la cara de mi jefe.

Levantarte para ir al curro, estar de cara al público, ponerte el uniforme, aguantar al típico pesao de turno, vamos, eso no está "ni pagao, ni agradecido" y lo que más me jode es que además te quita tiempo para hacer otras cosas.

Pero el curro también es una motivación, es una acumulación de experiencias, es conocer a otras personas. El curro te da la posibilidad de formarte a ti misma, sobre todo para las que no hemos estudiado. Y es que aunque a veces no nos guste nuestro curro, una vez que estás ahí, intentas pasártelo bien dentro de lo horrible que es trabajar. Nosotras somos muy trabajadoras, muy currantas, pero no siempre remuneradas, como pasa con las amas de casa.

Y es que no en todos los sitios se curra. Se curra en Carabanchel y se trabaja en el barrio de Salamanca. En la Castellana o en Chamartín la gente trabaja. Sin embargo en Pitis la gente curra, al igual que pasa en Usera o en Vallekas. Mi padre vive en Pozuelo, pero es albañil, así que curra. Y es que el curro no sólo va por zonas, sino que guarda relación con las personas, el tipo de curro que hagas y el ambiente en el que te muevas. El curro se usa, en unos sitios más y en otros menos.

En conclusión, que mañana curro. Así que no sé si podré quedar, pero vamos, que te llamo cuando salga.

\section{LÍNEA GRIS 1}

Algo pasa cada pocos minutos en la vieja estación de ferrocarril hoy convertida en centro comercial y en nudo de transportes. Si vienes de Moncloa y Argüelles, notarás que tu vagón también se ha renovado, que 
tus compañeros de viaje han cambiado antes de que llegues a Puerta del Ángel y a Alto de Extremadura. Ha sido en un abrir y cerrar de ojos, en un salir y entrar al túnel. En Príncipe Pío se han bajado unos y han subido otros. Estás en la Línea Gris, una línea que atraviesa el río justo en ese punto para iniciar su travesía por los barrios periféricos del sur, pero también en una línea gris -difusa, poco visible- que define los contornos de la desigualdad social en Madrid. A la gente "arreglada" y "cosmopolita" que viajaba contigo le ha sustituido otra gente con otros cuerpos más cansados, otros rostros más oscuros y otras ropas más baratas. Esa línea gris prosigue su recorrido con el mérito de conectar periferia con periferia -algo raro en una ciudad radial-, recorriendo Latina, Carabanchel y Usera antes de volver a cruzar el río y renovarse nuevamente tras su paso por Pacífico. Seguirá entonces los contornos del tercer cinturón haciendo el recorrido de las rondas, esas que delimitaban la ciudad burguesa del XIX, pero ya sin atreverse nuevamente a cruzar al otro lado, manteniéndose en la frontera. Sin embargo, más adelante volverá a cruzar el río, volverá a cruzar la frontera tras haber pasado nuevamente por Príncipe Pío. Porque la línea gris es la más larga del mundo, es infinita, no acaba en ningún sitio, da vueltas sobre la ciudad dibujando su composición social y atravesando las fronteras subjetivas. Es una línea que une distintos lugares de la ciudad, pero también una línea gris que nos separa a unos de otros.

\section{ENLACES DE INTERÉS:}

Carabancheleando: https://carabancheleando.net/ Video K-de-Kalle, Cine sin autor: https://www.cinesinautor.es/k-de-kalle

Diccionario de las Periferias , Editorial Traficantes de Sueños: https://www.traficantes.net/libros/diccio nario-de-las-periferias

\section{Notas}

1 La línea 6 del Metro de Madrid tiene un recorrido circular que transita gran parte de las periferias. Lleva el color gris. 